\title{
Clinical Characteristics of Hepatitis B Virus Patients After Switching to Tenofovir Alafenamide Fumarate: A Retrospective Observational Study
}

\author{
Abdullah S. Alghamdi ${ }^{1}$, Hammad S. Alothmani ${ }^{2}$, Mohammed Mogharbel ${ }^{2}$, Hazeez Albiladi Sr. ${ }^{2}$, \\ Mohamed Babatin ${ }^{2}$ \\ 1. Medical Department/Gastroenterology Unit, King Fahad General Hospital, Jeddah, SAU 2. Gastroenterology Unit, \\ King Fahad Hospital, Jeddah, SAU
}

Corresponding author: Abdullah S. Alghamdi, asgalghamdi2000@gmail.com

\section{Abstract \\ Background}

Hepatitis B Virus (HBV) continues to be a significant global health problem despite vaccination programs and effective antiviral drugs.

\section{Aim}

Assess tenofovir alafenamide fumarate (TAF) as a new treatment modality in light of the clinical characteristics of HBV patients.

\section{Settings and design}

A real-world observational study

\section{Methods and material}

We collected data of $71 \mathrm{HBV}$ patients and recorded the hepatitis B virus deoxyribonucleic acid (HBV-DNA) plasma levels and biochemistry test results for the alanine aminotransferase (ALT), aspartate aminotransferase (AST), and serum creatinine levels at three time points, including baseline, time of switching to TAF, and six months after switching.

\section{Results}

From the time of switching to TAF till six months later, HBV-DNA plasma levels significantly decreased from

Received 08/04/2020 Review began 08/04/2020 Review ended 09/07/2020 Published 09/11/2020

() Copyright 2020 Alghamdi et al. This is an open access article distributed under the terms of the Creative Commons Attribution License CC-BY 4.0., which permits unrestricted use, distribution, and reproduction in any medium, provided the original author and source are credited. 838.61 IU/mL to $16.7 \mathrm{IU} / \mathrm{mL}$ (p-value of <0.05). ALT and AST levels dropped from $29.05 \mathrm{U} / \mathrm{L}$ to $27 \mathrm{U} / \mathrm{L}$ and from $21.34 \mathrm{U} / \mathrm{L}$ to $20.7 \mathrm{U} / \mathrm{L}$ (p-values 0.328 and 0.410, respectively). Although TAF did not show a statistically significant reduction in the serum levels of AST, ALT, and creatinine, it showed a detectable maintenance level.

\section{Conclusions}

In the evaluated cohort, all clinical characteristics of HBV were maintained six months after switching patients to TAF.

Categories: Gastroenterology

Keywords: treatment of hbv, tenofovir alafenamide fumarate, retrospective study

\section{Introduction}

Hepatitis B virus (HBV) is a common blood-borne pathogen that continues to be a significant global health concern [1]. Despite effective vaccination programs, up to 250 million people around the world are still estimated to be positive for hepatitis B surface antigen (HBsAg) [2]. In the Eastern Mediterranean region, 3.3\% of the adult population are living with HBV, according to the World Health Organization (WHO) [3]. Nonetheless, owing to the improvement in preventive and care measures, the Kingdom of Saudi Arabia (KSA) witnessed a dramatic decline in the prevalence of chronic HBV infection from 8.3\% in 1988 to $1.3 \%$ in 2013 [4-5]. A chronic HBV infection is a major cause of morbidity and mortality, with almost 800,000 deaths annually. Without treatment, chronic hepatitis B can progress to cirrhosis, hepatocellular carcinoma (HCC), or liver failure [6]. Suppressive antiviral agents demonstrated a significant reduction in the risk of liverrelated complications and may delay or reverse the progression of the disease [7-8]. 
Tenofovir (TFV) is a nucleotide analog that inhibits the reverse transcription of HBV (NRTI) [9]. Tenofovir alafenamide fumarate (TAF) is a new prodrug of TFV that was designed to have greater plasma stability at a lower dose than tenofovir disoproxil fumarate (TDF), allowing successful delivery of the tenofovirdiphosphate (TFV-DP) to hepatocytes [10]. High systemic exposure to TFV in the form of TDF was associated with bone and renal toxicities [11]. Therefore, replacing TDF with TAF could reduce the circulating level of TFV by up to $90 \%[12]$.

Recent recommendations by both the European Association for the Study of Liver (EASL) and the American Association for the Study of Liver Diseases (AASLD) released in 2017 and 2018 included TAF as one of the first-line agents for HBV [13-14]. According to the recommendations released by the Saudi Association for the Study of Liver Diseases and Transplantation (SASLT) in 2014, the recommended anti-HBV agents in the country were entecavir (ETV) and TDF, along with lamivudine (LAM), telbivudine, adefovir, and pegylated interferon [15].

This observational study aimed to highlight the feasibility of TAF, after switching from other antiviral drugs including TDF, and its role in maintaining the clinical characteristics of HBV patients within the normal range.

\section{Materials And Methods Patients and methods}

Study Design

This study was a retrospective, observational cohort study conducted on HBV-infected patients from a single center in Saudi Arabia. All data were collected from patient records available between December 2006 and November 2017. We included records of patients who met the following criteria: (1) age $\geqslant 18$ years and $<80$ years; (2) a confirmed diagnosis of chronic HBV infection; (3) patients who received oral antiviral (OAV) treatment for at least six months ( \pm two weeks) prior to switching to TAF as per routine medical practice; and (4) patients attending the study site clinic with available patient records before and after switching to TAF. Patients were not eligible for this study if they fulfilled any of the following exclusion criteria: (1) incomplete data related to the variables of the primary endpoints; (2) creatinine clearance of $\leqslant 50 \mathrm{ml} / \mathrm{min}$, estimated by the Cockcroft-Gault formula [16]; (3) history of organ transplantation; (4) co-infection with hepatitis C virus, hepatitis D virus, or human immunodeficiency virus (HIV); (5) HCC; or (6) alcohol abuse.

This study followed the Strengthening the Reporting of Observational Studies in Epidemiology (STROBE) statement guidelines for the reporting of observational studies [17]. The protocol was reviewed and approved by the hospital ethical committee prior to study initiation. Informed consent was not required due to the retrospective nature of the study and considering that there was no associated risk. The data were collected anonymously; therefore, no patient identifier is revealed.

\section{Data collection}

Data were collected from the medical records of enrolled patients using an electronic case report form (CRF). Patient data were handled in compliance with all applicable privacy laws. Initial data collection was performed for each patient as per the schedule of events. Data were collected retrospectively at three time points: baseline (T1), at the time of the switch to TAF (T2), and at six $( \pm 0.5)$ months after switching to TAF (T3).

The study collected the following data at the three-time points: (1) demographic information: (age, gender, race); (2) relevant medical history, including the history of HBV diagnosis and management, and information regarding underlying diseases; (3) performed physical examination; any abnormal physical exam findings were documented; (4) body weight and body mass index (BMI); (5) vital signs (temperature, blood pressure, and pulse rate); (6) virology test results; HBV deoxyribonucleic acid (DNA) polymerase chain reaction (PCR) quantitative (HBV-DNA Quantitative by COBAS TaqMan (Roche Molecular Diagnostics Inc., Pleasanton, California) with a detection rate of less than $20 \mathrm{IU} / \mathrm{ML}$ ), hepatitis B envelope antigen (HBeAg; BEPIII machine from Siemens, Munich, Germany); (7) FibroScan (ECHOSENS Company, Paris, France) results that were classified according to the stage of fibrosis (F0-F4) [18]; (8) biochemistry test results; alanine transaminase (ALT), aspartate transaminase (AST), serum creatinine, serum albumin, total bilirubin, phosphorous, calcium, magnesium, and international normalized ratio (INR); (9) complete blood count test results; (10) abdominal ultrasound findings; (11) associated co-morbidities; and (12) any concomitant medications to be collected, including name, dose, and frequency.

\section{Statistical analysis}

Descriptive statistics were used to present the baseline demographic data and clinical characteristics at the three time points of the study. Categorical variables were presented by counts and percentages, whereas continuous variables were presented by mean and standard deviation, in case of the normal distribution, or by the median and interquartile range (IQR) otherwise. Numerical data were explored for normality using the 


\section{Cureus}

Kolmogorov-Smirnov test and the Shapiro-Wilk test. A paired t-test was used if the numeric data were normally distributed or non-parametric alternatives of the Wilcoxon signed-rank test if data were skewed. Categorical variables underwent a test of association using the McNemar test for paired data or Fisher's exact test. The significance level was two-sided, with a type 1 error of $5 \%$. The analyses were done using Statistical Package for Social Sciences version 24 (SPSS-24; IBM Corp., Armonk, New York).

\section{Results}

In this single-center study, a total of 77 subjects met the eligibility criteria; however, only 71 subjects were enrolled in the analysis. Two patients were removed from the analysis as they did not complete six months on the last antiviral medication before switching to TAF, and four patients were excluded due to missing information.

\section{Baseline characteristics of the study population}

The mean age of the study population was $45 \pm 12$ years. Most of the patients were Arabs, and both genders were represented equally (Table 1). Patients enrolled in the study were confirmed to be diagnosed with chronic HBV infection, with a mean disease duration of $13.65 \pm 6.5$ years. The mean HBV DNA levels at baseline were high (> 2x106 IU/mL) while most of the patients (88.7\%) were negative for HBeAg. The mean levels of AST, ALT, and serum creatinine were within the normal range of each parameter (Table 1). Baseline FibroScan results were not available in 39 of the 71 enrolled patients. Regarding the fibrosis stage at baseline, the majority of patients had F1 stage fibrosis (18.3\%), followed by F4 (12.7\%), F0 (8.5\%), F3 (8.5\%), and F2 (7.0\%). Liver ultrasound showed that more than half of the patients (53.5\%) had normal liver ultrasonography while approximately $20 \%$ of patients had the ultrasonographic features of fatty liver and $15.5 \%$ of patients had the ultrasonographic signs of cirrhosis (Table 1)

\section{Demographic variables}

Age, years (Mean \pm SD)

Gender (n, \%)

Male

Females

Race (n, \%)

Arab

non-Arab

$1(1.4 \%)$

Medical History

Present

Not present

$71(100 \%)$

Physical Examination (n, \%)

Normal

Abnormal

Body Mass Index (n, \%)

Underweight

Normal

Overweight

Obese

$22(31.0 \%)$

Virology

HBV DNA PCR quantitative (IU/ML) (Mean \pm SD)

$2190243.99 \pm 10151769.09$

HBeAg (n, \%)

Positive

Negative

$63(88.7 \%)$ 


\section{Cureus}

Biochemistry

ALT U/L (Mean \pm SD) [Normal range: 7 to $55 \mathrm{U} / \mathrm{L}]$

$49.66 \pm 98.16$

AST U/L (Mean \pm SD) [Normal range: 8 to $48 \mathrm{U} / \mathrm{L}$ ]

$31.12 \pm 42.92$

Serum Creatinine $(\mathrm{mg} / \mathrm{dL})($ Mean $\pm \mathrm{SD}$ ) [Normal range:0.84 to $1.21 \mathrm{mg} / \mathrm{dL}$ ]

$0.7014 \pm 0.277$

FibroScan

FO

F1

$13(18.3 \%)$

F2

$5(7.0 \%)$

F3

$6(8.5 \%)$

F4

$9(12.7 \%)$

Ultrasound

Normal

38 (53.5\%)

Fatty

$14(19.7 \%)$

Cirrhotic

$11(15.5 \%)$

\section{TABLE 1: Baseline characteristics of the study population}

Categorical data were presented by frequency and percentage; Continuous data were presented as mean \pm SD

HBV: hepatitis B virus; HBeAg: hepatitis B envelope antigen; ALT: alanine transaminase; AST: aspartate transaminase

\section{TAF feasibility}

The mean plasma HBV-DNA titer decreased from the time of the switch to six months later, from approximately $839 \mathrm{IU} / \mathrm{mL}$ to $17 \mathrm{IU} / \mathrm{mL}(\mathrm{p}=0.043)$ (Figure 1$)$.

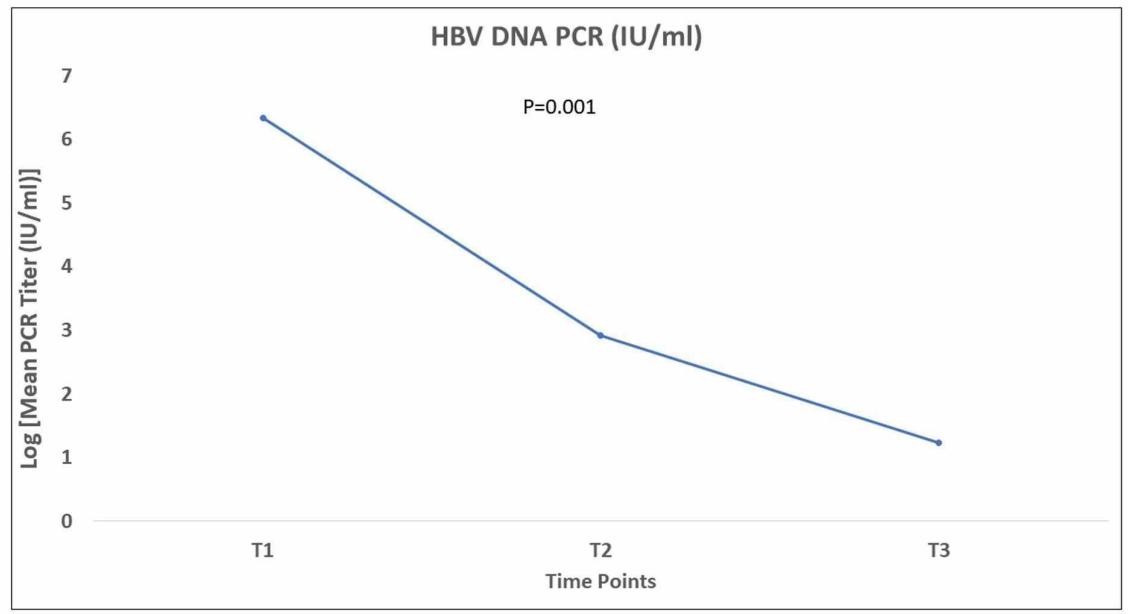

FIGURE 1: Mean HBV-DNA plasma level in $\log 10 \mathrm{IU} / \mathrm{mL}$ at the three time points of the study

HBV-DNA: hepatitis B virus deoxyribonucleic acid

The proportion of patients with positive HBeAg remained similar (12.7\%, p>0.05) at six months after switching to TAF (Figure 2). 


\section{Cureus}

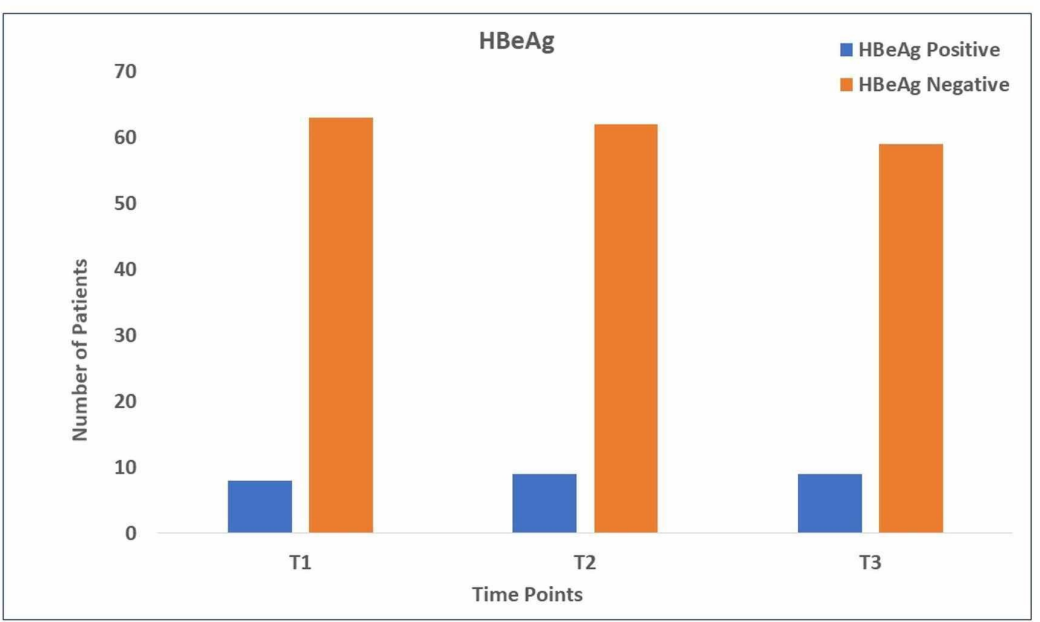

FIGURE 2: Numbers of HBeAg-positive and HBeAg-negative patients at the three time points of the study

HBeAg: hepatitis B envelope antigen

ALT and AST levels decreased slightly from the time of switching to TAF to six months later, but the changes were not significant $(\mathrm{p}=0.328$ and $\mathrm{p}=0.410$, respectively) (Figure $3 a$ ). Similar changes were observed for serum creatinine levels ( $\mathrm{p}=0.472$ ) (Figure $3 b$ ). 


\section{Cureus}
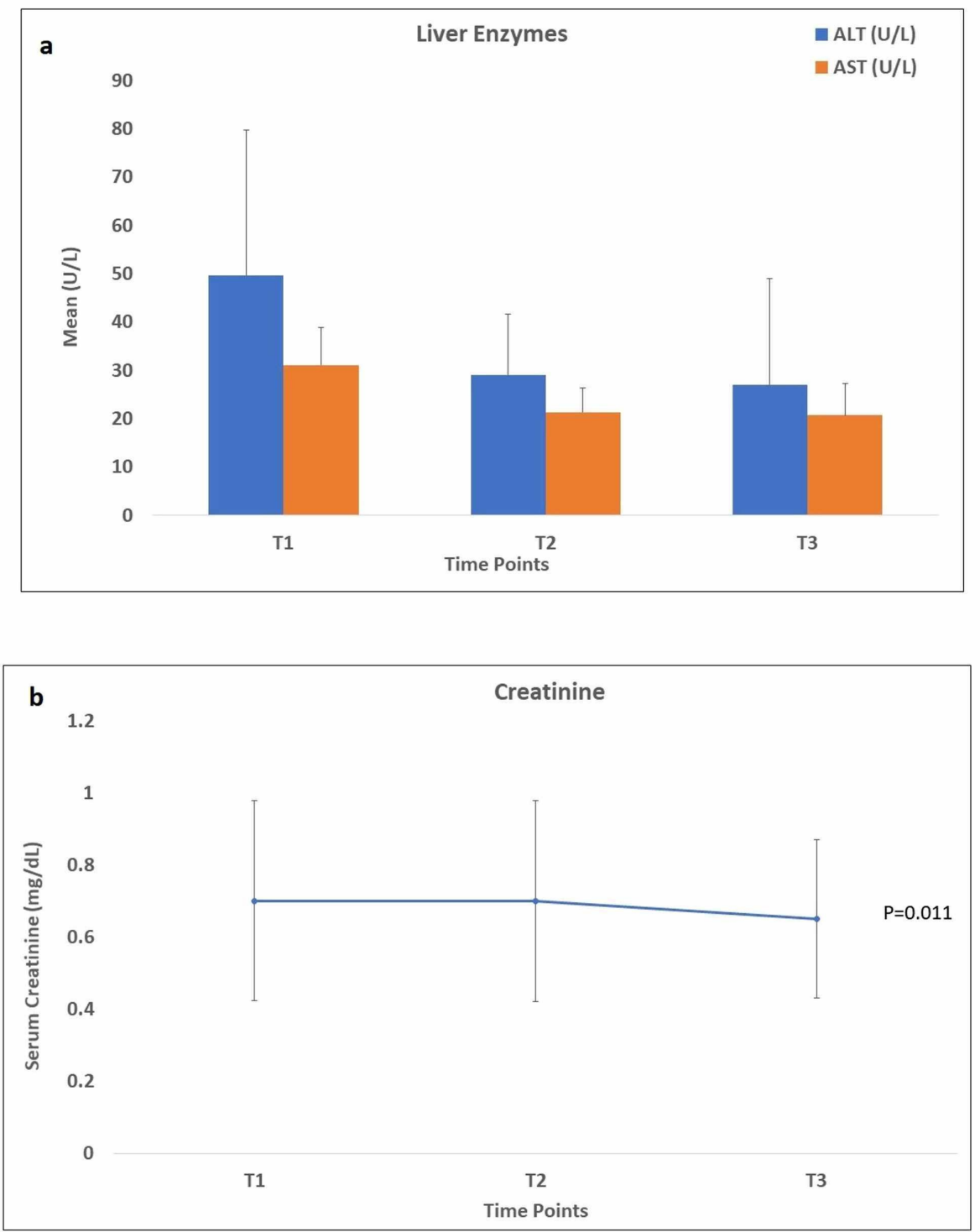

FIGURE 3: a) Mean levels of AST and ALT at the three time points of the study; b) Mean level of creatinine at the three time points of the study

ALT: alanine transaminase; AST: aspartate transaminase

On the other hand, FibroScan results and ultrasound showed different percentages for their corresponding categories and significant difference $(\mathrm{p}=0.001)$ between the baseline (time before switching) and the endpoint. When comparing FibroScan results and ultrasound variables between the switching point and the endpoint, they showed a non-significant difference $(\mathrm{p}=0.485$ and $\mathrm{p}=0.406$ ), respectively (Table 2 ). 


\section{Cureus}

\begin{tabular}{|c|c|c|c|c|c|}
\hline & T1 $(n=71)$ & T2 (n=71) & T3 $(n=70)^{a}$ & P-value* & P-value \\
\hline \multicolumn{6}{|c|}{ Fibrosis Score $(n, \%)$} \\
\hline F0 & $6(8.5 \%)$ & $3(4.2 \%)$ & $4(4.6 \%)$ & \multirow{5}{*}{0.001} & \multirow{5}{*}{0.485} \\
\hline F1 & 13(18.3\%) & $11(15.5 \%)$ & $8(11.3 \%)$ & & \\
\hline F2 & $5(7.0 \%)$ & $5(7.0 \%)$ & $3(4.2 \%)$ & & \\
\hline F3 & $6(8.5 \%)$ & $3(4.2 \%)$ & $3(4.2 \%)$ & & \\
\hline $\mathrm{F} 4$ & $9(12.7 \%)$ & $6(8.5 \%)$ & $5(7.0 \%)$ & & \\
\hline \multicolumn{6}{|c|}{ Ultrasound (n, \%) } \\
\hline Normal & $38(53.5 \%)$ & $32(45.1 \%)$ & $33(46.5 \%)$ & \multirow{3}{*}{0.001} & \multirow{3}{*}{0.406} \\
\hline Fatty & $14(19.7 \%)$ & 13(18.3\%) & $15(21.1 \%)$ & & \\
\hline Cirrhotic & $11(15.5 \%)$ & $11(15.5 \%)$ & $11(15.5 \%)$ & & \\
\hline
\end{tabular}

TABLE 2: Fibrosis score and ultrasound variables of HBV patients measured at three points in time

T1- 1st time point (At the baseline, on the last medication administered just before switching to TAF)

T2- 2nd time point (at the time of switch)

T3- 3rd time point $(6 \pm 0.5$ months after switching to TAF)

* For the comparison between the first and the third point in time (McNemar testing using binomial distribution)

${ }^{\star *}$ For the comparison between the second and the third point in time (McNemar testing using binomial distribution)

${ }^{a}$ One patient was excluded from this point due to the missing data

HBV: hepatitis B virus

The changes of several biochemical parameters tested at different time points are presented in Figure 4

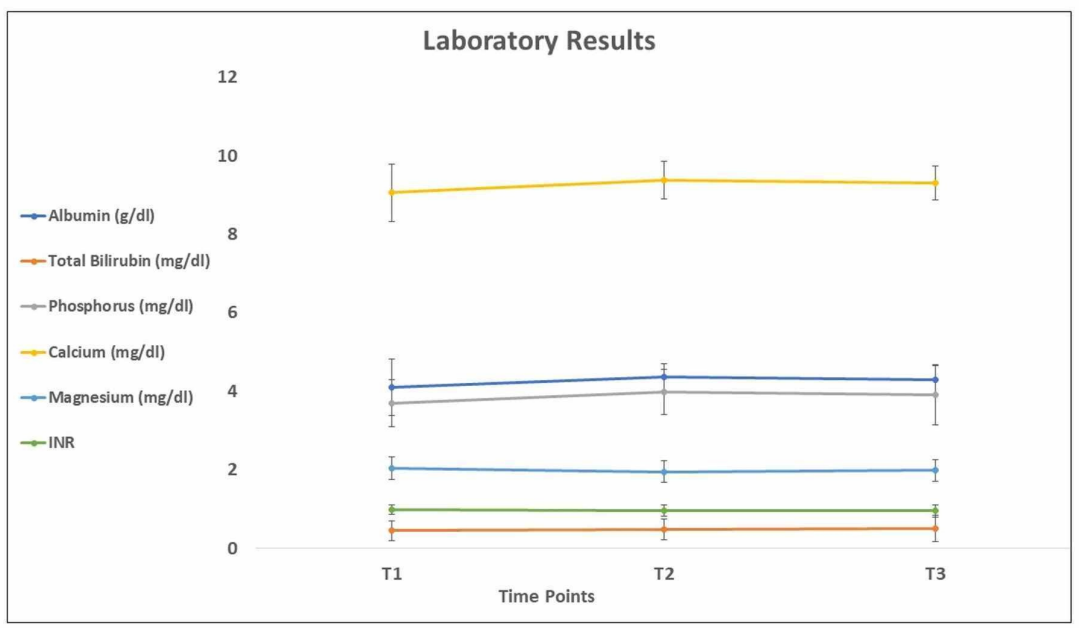

FIGURE 4: Biochemical parameters tested at the three time points of the study 
Mean albumin levels remained within the normal range (3.4-5.4g/dl); however, there was a non-significant increase $(\mathrm{p}=0.05)$ in the mean level from 4.11 at the baseline (T1) to 4.30 at the third point in time (T3). There was a significant difference between $\mathrm{T} 1$ and $\mathrm{T} 3 \mathrm{in}$ terms of phosphorus and calcium serum levels $(\mathrm{p}=0.02$ and $\mathrm{p}=0.013$, respectively). Regarding the magnesium, INR, and total bilirubin levels, there was no significant difference among the time points. The same was observed for the number of platelets, which was consistently within the normal range; however, there was a significant increase between the first and third points in time $(\mathrm{p}=0.004)$ (Figure 5).

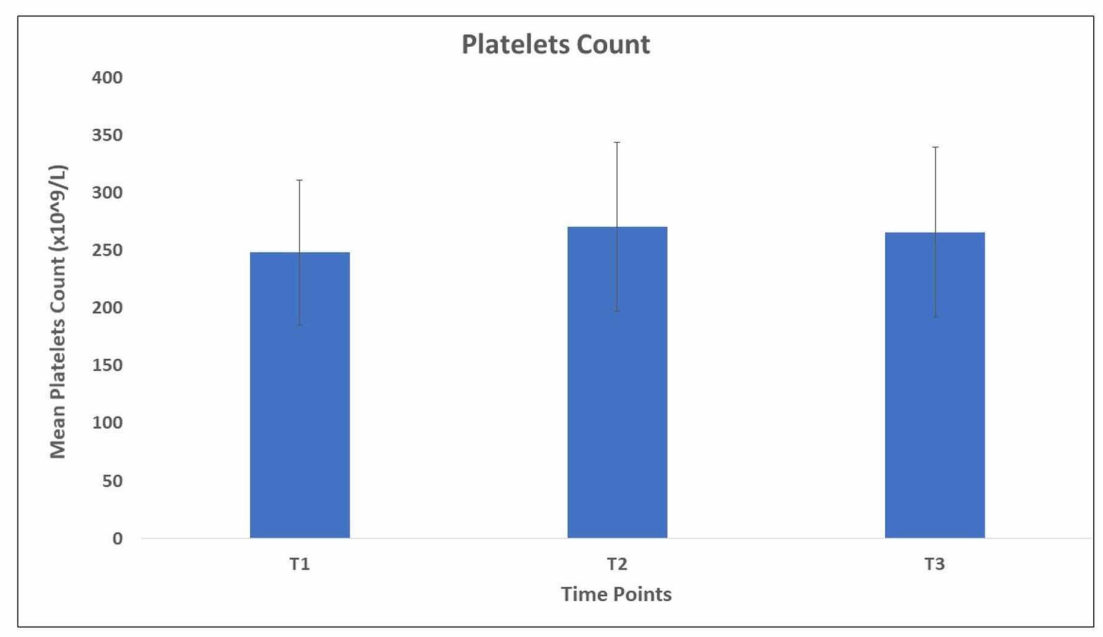

FIGURE 5: Mean platelets count at the three time points of the study.

\section{Reasons for switching to TAF - adverse events}

The top reason for switching to TAF was the TDF unavailability (81.7\%), followed by side effects from other HBV antiviral agents (14.1\%). Other reasons included the lack of efficacy of antiviral agents (1.4\%), safety concerns $(1.4 \%)$, and physician preference $(1.4 \%)$. There was no report of any adverse events due to medication across all study periods.

\section{Co-morbidities and concomitant medications}

Diabetes mellitus was present in $16.9 \%$ of the enrolled population, followed by hypertension in $9.9 \%$, hyperlipidemia and hypothyroidism in $4.2 \%$, and epilepsy in $1.4 \%$ of the patients. All co-morbidities were present at baseline (T1). Patients enrolled in the study were on multiple medications. The most frequently reported indications for concomitant medications were agents for diabetes mellitus management (21.1\%), followed by agents for hypertension (11.3\%) and benign prostatic hyperplasia (8.5\%). Other diseases requiring medications included hyperlipidemia, hypothyroidism, irritable bowel syndrome, urinary incontinence, and depression. The top medications taken by the patients were metformin $(\mathrm{n}=10)$, insulin $(n=4)$, tamsulosin $(n=4)$, amlodipine $(n=3)$, and esomeprazole $(n=3)$. Table 3 shows the medications taken by the patients with their corresponding dose, frequency, and route of administration. 


\begin{tabular}{|c|c|c|c|c|c|c|}
\hline Indication & Medication Name & Patients, $\mathbf{n}$ & Dose & Frequency & Route & Status \\
\hline \multirow{3}{*}{ Diabetes Mellitus } & Metformin & 10 & $500 \mathrm{mg}$ & Twice daily & Oral & Ongoing \\
\hline & Insulin & 4 & Variable & Twice daily & SC & Ongoing \\
\hline & Sitagliptin & 1 & $100 \mathrm{mg}$ & Once daily & Oral & Ongoing \\
\hline Hypothyroidism & Levothyroxine & 3 & Variable & Once daily & Oral & Ongoing \\
\hline \multirow{2}{*}{ Hyperlipidemia } & Rosuvastatin & 2 & $10 \mathrm{mg}$ & Once daily & Oral & Ongoing \\
\hline & Atorvastatin & 1 & $10 \mathrm{mg}$ & Once daily & Oral & Ongoing \\
\hline Irritable Bowel Syndrome & Mebeverine & 1 & 200 mg & Twice daily & Oral & Ongoing \\
\hline \multirow{6}{*}{ Hypertension } & Telmisartan & 1 & $40 \mathrm{mg}$ & Once daily & Oral & Ongoing \\
\hline & Indapamide & 1 & $1.5 \mathrm{mg}$ & Once daily & Oral & Ongoing \\
\hline & Amlodipine & 3 & $5 \mathrm{mg}$ & Twice daily & Oral & Ongoing \\
\hline & Metoprolol & 1 & $25 \mathrm{mg}$ & Twice daily & Oral & Ongoing \\
\hline & Captopril & 1 & $37.5 \mathrm{mg}$ & Three times & Oral & Ongoing \\
\hline & Perindopril & 1 & $2.5 \mathrm{mg}$ & Once daily & Oral & Ongoing \\
\hline Epilepsy & Valproic acid & 1 & $750 \mathrm{mg}$ & Once daily & Oral & Ongoing \\
\hline Peptic Ulcer Disease & Esomeprazole & 3 & $20 \mathrm{mg}$ & Twice daily & Oral & Ongoing \\
\hline \multirow{2}{*}{ Benign Prostatic Hyperplasia } & Tamsulosin & 4 & $4 \mathrm{mg}$ & Once daily & Oral & Ongoing \\
\hline & Finasteride & 2 & $5 \mathrm{mg}$ & Once daily & Oral & Ongoing \\
\hline Urinary Incontinence & Tolterodine & 1 & $2 \mathrm{mg}$ & Twice daily & Oral & Ongoing \\
\hline Depression & Escitalopram & 1 & $20 \mathrm{mg}$ & Twice daily & Oral & Ongoing \\
\hline
\end{tabular}

TABLE 3: Concomitant medication throughout the study period

\section{Discussion}

TAF targets OATP1B3 and ATP1B1 transporters, using them through passive diffusion, to enter the primary hepatocytes, where it is converted to TFV by carboxylesterase 1 [19]. The active metabolite of TFV, TFV-DP, which results from the phosphorylation of the intracellular TFV, inhibits the replication of HBV DNA through reverse transcriptase [20]. The stability of TAF and its ability to penetrate target cells reduces circulating TFV levels [21]. Consequently, the administration of lower effective TAF doses is allowed in comparison to its predecessor, TDF [22]. Moreover, TDF can be hydrolyzed in the gut and plasma, but TAF is not affected. In terms of drug interaction, no major drug interactions were observed in the case of TAF while in TDF, regimens containing ritonavir-boosted protease inhibitors were observed to increase the circulating levels of TDF and TDF-associated nephrotoxicity [22].

In this real-world observational study, we showed that six months of TAF therapy maintained the clinical stability of HBV-infected patients. The administration of TAF reduced HBV DNA plasma levels significantly $(p=0.043)$; however, it maintained ALT and AST levels within the normal range six months after switching to TAF without any significant change or increase in their levels. Moreover, the frequencies of FibroScan domains ( $F 0-F 4)$ were decreased significantly $(\mathrm{p}=0.001)$. Similarly, TAF maintained stable kidney function throughout the study duration, which was reflected by serum creatinine levels. These findings indicate that TAF can be a suitable substitute for the current antiviral regimens, including TDF in patients with HBV infection without loss of efficacy. Moreover, these higher ALT normalization rates can be translated into greater histological improvement with less inflammatory activity and reduced fibrosis progression, or a reduced risk of long-term complications such as HCC [23]. Our findings are in agreement with the results of the two-phase III multicenter randomized controlled trials comparing TAF and TDF reported by Buti et al. and Chan et al. [24-25]. Both studies showed that patients treated with TAF as compared to TDF achieved similar inhibition of HBV replication, which was maintained after switching patients from TDF to TAF while TAF offered higher ALT normalization rates before and after switching. There was no significant difference 
between the two drugs in terms of HBeAg loss or HBeAg seroconversion. However, another single-center study conducted on 48 chronic HBV patients compared patients who switched from entecavir to TAF and patients who continued on entecavir. This study reported that changes in the serum ALT levels and serum HBsAg levels were comparable between the two patient groups [26].

TAF, as compared to TDF, was shown to have favorable changes in serum creatinine levels and estimated glomerular filtration rate (eGFR) as well as in hip and spine bone marrow density (BMD) over the first two years of therapy $(p=0.001)$. Interestingly, both eGFR and BMD improved when patients were switched from TDF to TAF in those phase III trials [24-25]. BMD was not monitored in our study while we did not observe a significant improvement in serum creatinine levels after switching patients to TAF. In a study conducted on chronic HBV patients, Hagiwara et al. found that switching to TAF after entecavir or continuing on entecavir did not impact the renal function (estimated glomerular filtration rate (eGFR) levels) of both patient groups [26]. Our findings could be related to the different HBV agents instead of TDF only used by the patients before switching to TAF, the small number of patients included in our study increasing the possibility of a type II error and the relatively short duration of six months of TAF therapy. The European Association for the Study of the Liver (EASL) recommends TAF, instead of TDF, in patients with three specific risk factors: age $>60$ years, risk of bone disease, and renal problem [27]. As antiviral therapy is intended to last for a long time in the majority of chronic HBV patients who do not achieve HBsAg loss, it is reasonable to recommend to preferentially select drugs less prone to induce adverse bone and renal effects [28]. Therefore, all the above patients could benefit from the switch from TDF to TAF.

The availability of TAF and the side effects of the other HBV drugs seem to be the leading causes of switching chronic HBV patients to TAF in KSA. Blier et al. reported that switching medications during the management of the patient is a common practice; this switching may be due to the cost of previous medication or the availability of new medication with approved efficacy and improved safety [29]. Regarding safety, TAF showed a promising profile with no recorded drug-related adverse events during six months of therapy, although it was used in patients with several co-morbidities who were using many concomitant medications. In terms of pregnancy, TAF is classified as FDA pregnancy category B drugs, as no human data about the use of this drug in pregnancy is available [23]. All co-morbidities were already present at the onset of the last HBV therapy before TAF and none developed during the administration of TAF. In contrast, other studies reported some adverse drug reactions (ADRs) such as nausea, back pain, fatigue, and headache [24-25], which, however, were not related to the study drug. None of our patients discontinued TAF, whereas only $1 \%$ of patients discontinued treatment with TAF as compared to $1.2 \%$ in the TDF group in the phase III TAF registrational trials [24-25]. All these data indicate that TAF is a well-tolerated therapeutic agent [24].

Our study has some limitations: (1) a selection bias can be identified, as all patients were selected from one center and there was no representation of other races in Saudi Arabia; (2) the relatively small sample size of the study population, which can be explained by our restricting criteria and conducting the study in a singlecenter; (3) the high rate of missing records, including adverse events, which hindered drawing useful conclusions in some of the variables; (4) although patients with poor renal function would have been useful to include in this study; however, we could not find enough data to be considered.

\section{Conclusions}

Our findings suggest that TAF is a feasible, well-tolerated, and safe alternative in chronic HBV patients who are treated with TDF, ETV, LAM, and adefovir. TAF maintained significant viral suppression and higher AST and ALT normalization rates. The advantage of TAF in the reduction of serum HBsAg levels should be further evaluated, especially in patients with HBV infection without underlying cirrhosis.

\section{Additional Information \\ Disclosures}

Human subjects: Consent was obtained by all participants in this study. Ministry of Health, King Fahd Medical City issued approval FWA00018774. I am pleased to inform you that your submission dated September 11, 2019, for the study titled "Clinical Characteristics of Hepatitis B Virus Patients after Switching to Tenofovir Alafenamide Fumarate: A Retrospective Observational Study" was reviewed and approved according to Good Clinical Practice Guidelines. Animal subjects: All authors have confirmed that this study did not involve animal subjects or tissue. Conflicts of interest: In compliance with the ICMJE uniform disclosure form, all authors declare the following: Payment/services info: Dr. Abdullah Alghamdi has received fees as a member of advisory committees and as an invited speaker from Gilead Sciences, Merck Sharpe Dohme, and AbbVie. Dr. Mohammed Babatin has received fees as a member of advisory committees and as an invited speaker from Gilead Sciences, Merck Sharpe Dohme, and AbbVie. Data collection, analysis, manuscript writing, and publication of this study were funded by Gilead Sciences Inc. Financial relationships: All authors have declared that they have no financial relationships at present or within the previous three years with any organizations that might have an interest in the submitted work. Other relationships: All authors have declared that there are no other relationships or activities that could appear to have influenced the submitted work. 


\section{Acknowledgements}

We would like to thank Prof. Georgios Papatheodoridis for his significant contribution in reviewing and revising this manuscript.

\section{References}

1. Kwon SY, Lee CH: Epidemiology and prevention of hepatitis B virus infection . Korean J Hepatol. 2011, 17:87-95. 10.3350/kjhep.2011.17.2.87

2. Nicolini LA, Orsi A, Tatarelli P, Viscoli C, Icardi G, Sticchi L: A global view to HBV chronic infection: evolving strategies for diagnosis, treatment and prevention in immunocompetent individuals. Int J Environ Res Public Health. 2019, 16:3307. 10.3390/ijerph16183307

3. Jefferies M, Rauff B, Rashid H, Lam T, Rafiq S: Update on global epidemiology of viral hepatitis and preventive strategies. World J Clin Cases. 2018, 6:589-599. 10.12998/wjcc.v6.i13.589

4. Al-Faleh FZ: Hepatitis B infection in Saudi Arabia . Ann Saudi Med. 1988, 8:474-480. 10.5144/02564947.1988.474

5. Alswaidi FM, Memish ZA, Al Hakeem RF, Atlam SA: Saudi Arabian expatriate worker fitness-screening programme: a review of 14 years of data. East Mediterr Heal J. 2013, 19:664-670.

6. Di Bisceglie AM: Hepatitis B and hepatocellular carcinoma . Hepatology. 2009, 49:56-60. 10.1002/hep.22962

7. Giulea C, Enciu O, Bîrcă T, Miron A: Selective intraoperative cholangiography in laparoscopic cholecystectomy. Chirurgia. 2016, 111:26-32.

8. Lu L-G: Antiviral therapy of liver cirrhosis related to hepatitis B virus infection . J Clin Transl Hepatol. 2014, 2:197-201. 10.14218/JCTH.2014.00022

9. Jones SA, Murakami E, Delaney W, Furman P, Hu J: Noncompetitive inhibition of hepatitis B virus reverse transcriptase protein priming and DNA synthesis by the nucleoside analog clevudine. Antimicrob Agents Chemother. 2013, 57:4181-4189. 10.1128/AAC.00599-13

10. De Clercq E: Tenofovir alafenamide (TAF) as the successor of tenofovir disoproxil fumarate (TDF) . Biochem Pharmacol. 2016, 119:1-7. 10.1016/j.bcp.2016.04.015

11. Byrne R, Carey I, Agarwal K: Tenofovir alafenamide in the treatment of chronic hepatitis B virus infection: rationale and clinical trial evidence. Therap Adv Gastroenterol. 2018, 11: 10.1177/1756284818786108

12. Ruane PJ, Dejesus E, Berger D, et al.: Antiviral activity, safety, and pharmacokinetics/pharmacodynamics of tenofovir alafenamide as 10-day monotherapy in HIV-1-positive adults. Journal of Acquired Immune Deficiency Syndromes. 2013, 63:449-455.

13. Terrault NA, Lok ASF, McMahon BJ, et al.: Update on prevention, diagnosis, and treatment of chronic hepatitis B: AASLD 2018 hepatitis B guidance. Hepatology. 2018, 67:1560-1599. 10.1002/hep.29800

14. Idilman R: The summarized of EASL 2017 clinical practice guidelines on the management of hepatitis B virus infection. Turkish J Gastroenterol. 2017, 28:412-416. 10.1016/j.jhep.2017.03.021

15. Al-Hamoudi W, Abaalkhail F, Elsiesy H, Alghamdi MY, Alalwan A, AlMasri N, Al-Hamoudi W: SASLT practice guidelines for the management of hepatitis B virus. Saudi J Gastroenterol. 2014, 20:5-25. 10.4103/1319-3767.126311

16. Cockcroft DW, Gault H: Prediction of creatinine clearance from serum creatinine . Nephron. 1976, 16:31-41. 10.1159/000180580

17. Elm E von, Altman DG, Egger M, Pocock SJ, Gøtzsche PC, Vandenbroucke JP: Strengthening the reporting of observational studies in epidemiology (STROBE) statement: guidelines for reporting observational studies. BMJ. 2007, 335:806-808. 10.7326/0003-4819-147-8-200710160-00010

18. Castera L, Yuen Chan HL, Arrese M, et al.: EASL-ALEH clinical practice guidelines: non-invasive tests for evaluation of liver disease severity and prognosis. J Hepatol. 2015, 63:237-264.

19. Vemlidy (tenofovir alafenamide). (2013). https://search.proquest.com/openview/1156ede5cbe165d5af92d8e1a430b0d4/1?cbl=136155\&pqorigsite $=$ gscholar\&utm_campaign $=\ldots$...

20. Delaney WE, Ray AS, Yang H, et al.: Intracellular metabolism and in vitro activity of tenofovir against hepatitis B virus. Antimicrob Agents Chemother. 2006, 50:2471-2477. 10.1128/AAC.00138-06

21. Snopková S, Havlíčková K, Husa P: Tenofovir alafenamide fumarate - a new generation of tenofovir [Article in Czech]. Klin Mikrobiol Infekc Lek. 2016, 22:111-117.

22. Ray AS, Fordyce MW, Hitchcock MJM: Tenofovir alafenamide: a novel prodrug of tenofovir for the treatment of human immunodeficiency virus. Antiviral Res. 2016, 125:63-70. 10.1016/j.antiviral.2015.11.009

23. Viganò M, Loglio A, Grossi G, Lampertico P: Tenofovir alafenamide (TAF) treatment of HBV, what are the unanswered questions?. Expert Rev Anti Infect Ther. 2018, 16:153-161. 10.1080/14787210.2018.1428561

24. Buti M, Gane E, Seto WK, et al.: Tenofovir alafenamide versus tenofovir disoproxil fumarate for the treatment of patients with HBeAg-negative chronic hepatitis B virus infection: a randomised, double-blind, phase 3, non-inferiority trial. Lancet Gastroenterol Hepatol. 2016, 1:196-206. 10.1016/S24681253(16)30107-8

25. Chan HLY, Fung S, Seto WK, et al.: Tenofovir alafenamide versus tenofovir disoproxil fumarate for the treatment of HBeAg-positive chronic hepatitis B virus infection: a randomised, double-blind, phase 3, noninferiority trial. Lancet Gastroenterol Hepatol. 2016 Nov, 1:185-95. 10.1016/S2468-1253(16)30024-3

26. Hagiwara S, Nishida N, Ida H, et al.: Switching from entecavir to tenofovir alafenamide versus maintaining entecavir for chronic hepatitis B. J Med Virol. 2019, 91:1804-1810. 10.1002/jmv.25515

27. Lampertico P, Agarwal K, Berg T, et al.: EASL 2017 clinical practice guidelines on the management of hepatitis B virus infection. J Hepatol. 2017, 67:370-398. 10.1016/j.jhep.2017.03.021

28. Chan HL-Y, Buti M, Flisiak R, et al.: IDDF2018-ABS-0107 efficacy and safety of tenofovir alafenamide (TAF) at 96 weeks in chronic HBV (CHB) patients with risk factors for use of tenofovir disoproxil fumarate (TDF). Gut. Ltd and British Society of Gastroenterology; 2018. p, 67:A97. 10.1136/gutjnl-2018-IDDFabstracts.208

29. Blier P, Margolese HC, Wilson EA, Boucher M: Switching medication products during the treatment of 


\section{Cureus}

psychiatric illness. Int J Psychiatry Clin Pract. 2019, 23:2-13. 10.1080/13651501.2018.1508724 